\title{
Aspectos fisiopatológicos do COVID-19 e uso de ventilação não invasiva. É possível?
}

\author{
Pathophysiological aspects of COVID-19 and \\ use of non-invasive ventilation. Is it possible?
}

\author{
Jaqueline Aparecida Almeida Spadari' (1) \\ Giulliano Gardenghi ${ }^{2}$ (1)
}

\begin{abstract}
${ }^{1}$ Hospital e Maternidade São Cristóvão (São Paulo). São Paulo, Brasil. jaque.spadari@gmail.com ${ }^{2}$ Autor para correspondência. Faculdade CEAFI (Goiânia), Hospital Encore (Aparecida de Goiânia), Hospital e Maternidade São Cristóvão (São Paulo). Goiás, São Paulo, Brasil. ggardenghi@encore.com.br
\end{abstract}

De acordo com a Organização Mundial de Saúde (OMS), em junho de 2020 os casos confirmados do COVID-19 causados pelo coronavírus (SARsCOV-2) já haviam ultrapassado sete milhões pelo mundo. Declarado estado de pandemia em 11 de março de 2020, as equipes de saúde se deparam com planos estratégicos de condutas variáveis ${ }^{1}$ especialmente na indicação e no manuseio do suporte ventilatório não invasivo.

A taxa de letalidade dos infectados é determinada pela combinação de características intrínsecas (comorbidades, idade, sistema imunológico) e oferta/disponibilidade de recursos terapêuticos² ${ }^{2}$. No entanto, ao considerarmos a mortalidade em hospitalizados em uma série de 5.700 casos admitidos em 12 hospitais de Nova York foi observado que a faixa etária entre 20 e 50 anos é maior do que a esperada com aumento de $10 \%$ em pessoas entre 20 e 30 anos $^{3}$. Além disso, a doença apresenta características mais brandas na primeira semana que se agravam posteriormente, com óbito aumentando exponencialmente entre $o 12^{\circ}$ e $24^{\circ}$ dia, descaracterizando um processo agudo 4 .
As comorbidades se associam proporcionalmente à gravidade dos desfechos. Na tentativa de facilitar o manejo clínico e fazer uma estratificação de risco, um grupo de pesquisadores chineses elaborou o CALL Score, que calcula conforme as comorbidades, idade, valor dos linfócitos e LDH (lactato desidrogenase). Um score abaixo de 6 indica probabilidade menor de evolução enquanto superior a 9 pontos, o contrário ${ }^{5}$. Foi demonstrado pior prognóstico em pacientes hospitalizados com hipertensão, diabetes e doença coronariana, idade avançada, score SOFA (Sequential Organ Failure Assessment) elevado (disfunção orgânica) e valores elevados de D dímero. O SARS-CoV-2 foi detectável até o óbito e em sobreviventes, sendo sua duração mais longa de 37 dias. A mediana de liberação do vírus nas vias aéreas foi de, no mínimo, 14 dias, podendo chegar a 60 dias ${ }^{6,1}$.

O aumento da letalidade estimula a busca por meios de tratamento eficazes da infecção. Uma série de indicações e contra indicações vem sendo aplicadas no manejo desses pacientes, especialmente em relação ao uso da ventilação não invasiva (VNI). 
Esse editorial busca abordar as dúvidas a respeito da transmissão do vírus, das formas de precaução e o quanto esses fatores podem influenciar a aplicação da VNI.

O uso da VNI pode promover melhora da oxigenação e da saturação periférica de oxigênio, diminuição do trabalho respiratório e redução significativa na necessidade de intubação e da mortalidade, quando aplicada com os critérios de segurança, da maneira apropriada e monitorização contínua. Em uma amostra de 138 hospitalizados foi observado que a dispneia se inicia no $5^{\circ}$ dia pós infecção, a internação no $7^{\circ}$ e a SDRA no $8^{\circ}$. Na UTI são aplicados oxigenoterapia de alto fluxo, VNI e ventilação mecânica invasiva ${ }^{8}$. No entanto, persistem dúvidas sobre quando usar a VNI em casos confirmados de COVID-19. O ponto chave de todas as discussões sobre o uso da ventilação mecânica, seja ela invasiva ou não, gira em torno do alto risco de contaminação pelo vírus.

Em nota oficial, a Agência Nacional de Vigilância Sanitária (ANVISA) criou orientações específicas sobre o manejo e descarte dos resíduos utilizados e principalmente, a importância da higienização das mãos e o uso correto dos equipamentos de proteção individual (EPIs) pelos profissionais de saúde, sendo eles: gorro, óculos de proteção ou protetor facial, máscara (cirúrgica ou de proteção respiratória - n95, conforme o procedimento realizado), avental impermeável de mangas longas e luvas de procedimento? .

Procedimentos geradores de aerossóis podem aumentar o contágio e incluem intubação orotraqueal, VNI e ventilação manual pós intubação. Aspiração traqueal, broncoscopia, uso de nebulizadores, administração de oxigenoterapia, cânula nasal de alto fluxo, manipulação da máscara (oxigenoterapia ou interfaces para VNI), desfibrilação, compressões torácicas, inserção de sonda nasogástrica e coleta de secreção pulmonar. A transmissão mais consistente está fortemente relacionada a intubação orotraqueal ou traqueostomia, devido a necessidade de proximidade por um tempo prolongado com a via aérea do paciente. Os dados encontrados a respeito da contaminação durante a aplicação de VNI, não foram suficientemente fortes para estabelecerem certezas que justifiquem o veto ao procedimento ${ }^{10}$. Amostras de RNA do vírus SARSCoV-2 foram encontradas em mobiliários do quarto, itens pessoais e corredor por dispersão de partículas. Os resultados nos levam a ponderar sobre o potencial de contaminação do material genético encontrado nos aerossóis, uma vez que nos casos citados, as amostras não apresentaram crescimento em culturas ${ }^{11}$.

No primeiro caso COVID-19 confirmado na Califórnia, 121 profissionais mantiveram contato com o paciente, destes, 43 desenvolveram sintomas durante 14 dias após exposição e três tiveram resultado confirmado de infecção, apesar da não utilização dos EPIs preconizados. Em outro hospital, dos 146 profissionais envolvidos na assistência de contaminados, nenhum se infectou, apesar do uso de EPIs para atendimento de paciente em ventilação invasiva com circuito fechado de aspiração desde a sua admissão. Estes achados nos levam a crer na eficiência das barreiras em evitar contaminação ${ }^{12}$. Um outro caso de um paciente chinês em $\mathrm{VNI}$, que testou positivo para SARS-CoV-2 no momento e após a extubação chama a atenção. Mais de 35 profissionais estiveram envolvidos em procedimentos de aerossóis, utilizando máscara cirúrgica, não a máscara n95, apontada para o uso nessas situações. Todos foram acompanhados e nenhum deles apresentou sintomas ou resultados positivos nas coletas de swab orofaríngeo ${ }^{13}$. Isso nos leva a concluir que ainda há necessidade de estudos mais contundentes sobre a contaminação de equipes de saúde.

Em abril, foi divulgado na The Lancet um questionamento interessante acerca desse assunto, apontando inicialmente que o medo da alta transmissibilidade faz as equipes excluírem a possibilidade de medidas ventilatórias não invasivas, e ainda, levanta a pertinência de abdicar do recurso não invasivo como prática em um cenário de recursos limitados ${ }^{14}$. Inclusive, é importante citar que logo após declarado o estado de pandemia, a OMS disponibilizou um Guideline no qual não restringem, mas consideram o uso de VNI em uma população bem selecionada dos pacientes com quadro de hipoxemia ${ }^{14,15}$.

A maior preocupação dos profissionais de saúde frente a VNI nos casos confirmados de COVID-19 é o uso do circuito aberto de ventilação. Nesses casos, assume-se vazamento de ar, intencional ou não, ocasionado tanto pela necessidade do uso da válvula exalatória no circuito, quanto pela fixação da máscara no rosto do paciente, aumentando assim, a disseminação do vírus no ambiente, devido à dispersão de aerossóis. Partindo desse princípio, as recomendações sugerem que os pacientes estejam em precaução de contato, gotículas e aerossóis; alocados preferencialmente - não obrigatoriamente - em quartos com pressão negativa, uma vez que esses atuam 
reduzindo a chance de infecção, já que a pressão interna deste cômodo permanecerá menor do que a externa, impedindo as correntes de ar ${ }^{16,17}$.

Na tentativa de atenuar uma possível aerossolização, deve-se atentar aos detalhes da montagem do circuito do ventilador, optando preferencialmente por circuitos de ramo duplo e filtros antibacterianos/antivirais de alta eficiência (tanto no ramo expiratório do ventilador mecânico quanto proximal ao paciente). Quanto às interfaces disponíveis, o bom ajuste também minimiza a dispersão generalizada do ar expirado14-16. Há predileção pela indicação do capacete Helmet ${ }^{\circledR}$ (máscara não ventilada), que pode reduzir a possibilidade de contaminação. No entanto, pacientes em uso de VNI separados em grupos foram avaliados quanto à eficácia da máscara facial convencional comparada a interface Helmet ${ }^{\circledR}$ (capuz transparente que cobre a cabeça do paciente e tem uma gola de borracha no pescoço). Houve redução significativa nas taxas de intubação e mortalidade no grupo com capacete. Vale destacar que o uso do capacete proporciona redução de vazamento e permite ajustes mais elevados de pressão positiva expiratória final (PEEP), potencializando o recrutamento alveolar e diminuindo o trabaIho respiratório ${ }^{17-20}$. No entanto, a máscara facial total também apresenta mínimo vazamento e permite maior pressurização das vias aéreas ${ }^{21}$.

$\mathrm{Na}$ Espanha, pesquisadores elaboraram um consenso clínico com recomendações sobre suporte respiratório não invasivo (VNI e oxigenoterapia com cateter nasal de alto fluxo) no paciente adulto com insuficiência respiratória aguda secundária a SARS-CoV-2 na tentativa de otimizar o suporte clínico, auxiliar a identificar a indicação e descrever os processos de alto risco de falha da VNI, para que sejam evitados. Os resultados sugerem aplicação da VNI pacientes com $\mathrm{PaO} 2 / \mathrm{FiO} 2$ superior a 100 e sem falência de múltiplos órgãos (score de gravidade APACHE inferior a 20). O uso da VNI deve ser realizado após avaliação criteriosa por no mínimo 30 minutos, não excedendo 60 minutos nesses casos. Caso o paciente não apresente melhora clínica evidente, como diminuição do desconforto ventilatório, recomenda-se descontinuar a tentativa de suporte não invasivo, especialmente caso o paciente evolua com volume minuto superior a $10 \mathrm{l} / \mathrm{min}$, volume corrente acima de $9 \mathrm{ml} / \mathrm{Kg}$ predito, frequência respiratória acima de 25ipm, necessidade de pressões positivas expiratórias final superiores a $10 \mathrm{cmH} 2 \mathrm{O}$ ou necessidade de $\mathrm{FiO} 2$ elevada (elevada a 50\%). Esse conjunto de sinais demonstram gravidade que indicam neces- sidade de ventilação invasiva. Segundo as Diretrizes Brasileiras de Ventilação Mecânica, em população hipoxêmica, o índice de sucesso da VNI para evitar intubação orotraqueal e demais complicações, pode ser de $50 \% \frac{16,21,22}{}$. Já outro estudo sugere que a tentativa de VNI deve ser indicada quando a relação $\mathrm{PaO} 2 / \mathrm{FiO}_{2}$ estiver maior ou igual a $150 \mathrm{mmHg}^{23}$.

Alguns estudos documentam a viabilidade de realizar a VNI em pacientes de COVID-19 com bons resultados nos que atenderam aos critérios do teste e uso da interface com SDRA de graus leve e moderado submetidos por cinco dias a tratamento com VNI associada a posição prona. Houve melhora significante da saturação periférica de oxigênio, aumento da relação de oxigenação e diminuição da frequência respiratória. Trata-se de uma sugestão terapêutica a ser considerada, onde foi observada resposta positiva em $80 \%$ dos pacientes 2 . Recentemente, uma metanálise que incluiu 25 estudos envolvendo 3.804 pacientes com insuficiência respiratória hipoxêmica aguda, sugere a interface Helmet ${ }^{\circledR}$ como mais vantajosa. O uso da VNI foi capaz de reduzir mortalidade e intubação orotraqueal, quando comparada as outras interfaces de administração de oxigenoterapia 20 .

Ainda são necessários estudos controlados e randomizados, revisões sistemáticas com metanálise e evidências mais robustas, mas a VNI, em alguns casos confirmados de COVID-19 vêm demonstrando bons resultados na prática clínica em diversos países, inclusive no Brasil, em relatos de fisioterapeutas que estão na linha de frente. O emprego da VNI e o uso correto dos EPIs são fundamentais para se evitar contaminação da equipe assistente. Em suma, a VNI não deve ser encarada como uma restrição absoluta, inclusive, parece ser uma boa indicação de tratamento, especialmente em um cenário de poucos recursos, desde que realizada com segurança, monitorização criteriosa e não postergando a indicação da intubação orotraqueal.

\section{Referências}

1. World Health Organization. Coronavirus disease (COVID-19) outbreak. [Internet]. 2020. Disponível em: https://www.who.int/ emergencies/diseases/novel-coronavirus-2019

2. Souza CDF, Paiva JPS, Leal TC, Silva LF, Santos LG. Evolução espaçotemporal da letalidade por COVID-19 no Brasil. J Bras Pneumol. 2020;46(4):e20200208. doi: 10.36416/1806-3756/ e20200208 
3. Richardson S, Hirsch JS, Narasimhan M, Crawford JM, McGinn T, Davidson KW et al. Presenting Characteristics, Comorbidities, and Outcomes Among 5700 Patients Hospitalized With COVID-19 in the New York City Area. JAMA. 2020;323(20):2052-2059. doi: 10.1001/jama.2020.6775

4. Ruan Q, Yang K, Wang W, Jiang L, Song J. Clinical predictors of mortality due to COVID-19 based on an analysis of data of 150 patients from Wuhan, China. Intensive Care Med. 2020;46(5):846848. doi: 10.1007/s00134-020-05991-x

5. Ji D, Zhang D, Xu J, Chen Z, Yang T, Zhao P et al. Prediction for Progression Risk in Patients with COVID-19 Pneumonia: the CALL Score. Clinical Infectious Diseases. 2020. doi: 10.1093/cid/ciaa414

6. Zhou F, Yu T, Du R, Fan G, Liu Y, Liu Z et al. Clinical course and risk factors for mortality of adult inpatients with COVID-19 in Wuhan, China: a retrospective cohort study [published correction appears in Lancet. 2020;395(10229):1038]. Lancet. 2020;395(10229):1054-1062. doi:10.1016/S0140-6736(20)30566-3

7. Zou L, Ruan F, Huang $M$, Liang L, Huang $H$, Hong $Z$ et al. SARSCoV-2 Viral Load in Upper Respiratory Specimens of Infected Patients. N Engla J Med. 2020; 382:1177-1179. doi: $10.1056 /$ NEJMc2001737

8. Wang D, Hu B, Hu C, Zhu F, Liu X, Zhang J et al. Clinical Characteristics of 138 Hospitalized Patients With 2019 Novel Coronavirus-Infected Pneumonia in Wuhan, China. JAMA. 2020;323(11):1061-1069. doi: 10.1001/jama.2020.1585

9. Ministério da Saúde. Saúde e segurança do trabalhador (EPI). [Internet]. 2020. Disponível em: https://coronavirus.saude.gov.br/ saude-e-seguranca-do-trabalhador-epi

10. Tran K, Cimon K, Severn M, Pessoa-Silva CL, Conly J. Aerosol generating procedures and risk of transmission of acute respiratory infections to healthcare workers: a systematic review. PLoS One. 2012;7(4):e35797. doi: 10.1371/journal.pone.0035797

11. Santarpia JL, Rivera DN, Herrera V, Morwitzer MJ, Creager H, Santarpia GW et al. Transmission Potential of SARS-CoV-2 in Viral Shedding Observed at the University of Nebraska Medical Center. MedRxiv. 2020. doi: 10.1101/2020.03.23.20039446

12. Heinzerlin A, Stuckey MJ, Scheuer T, Xu K, Perkins KM, Resseger $\mathrm{H}$ et al. Transmission of COVID-19 to Health Care Personnel During Exposures to a Hospitalized Patient - Solano County, California, February 2020. MMWR Morb Mortal Mkly Rep. 2020; 69(15):472-476. doi: 10.15585/mmwr.mm6915e5

13. Kangqi NG, Poon BH, Puar THK, Quah JLS, Loh WJ, Wong YJ et al. COVID-19 and the Risk to Health Care Workers: A Case Report. Ann Intern Med. 2020;172(11):766-767. doi: 10.7326/L20-0175

14. Arulkumaran N, Brealey D, Howell D, Singer M. Use of noninvasive ventilation for patients with COVID-19: a cause for concern? Lancet Respir Med. 2020;8(6):45. doi: 10.1016/S22132600(20)30181-8
15. World Health Organization. Clinical management of severe acute respiratory infection (SARI) when COVID-19 disease is suspected: interim guidance, 13 March 2020. [Internet]. 2020. Disponível em: https://apps.who.int/iris/handle/10665/331446

16. Gómez CC, Rodríguez OP, Torné ML, Santaolalla CE, Jiménez JFM, Fernández JG et al. “Clinical Consensus Recommendations Regarding Non-Invasive Respiratory Support in the Adult Patient with Acute Respiratory Failure Secondary to SARSCoV-2 infection.". Arch Bronconeumol. 2020;56 Suppl (2):11-18. doi:10.1016/j.arbres.2020.03.005

17. Garcés HH, Muncharaz AB, Crespo RZ. Ventilación mecánica no invasiva y COVID-19. Minimizando la dispersión. Med Intensiva. 2020. doi: 10.1016/j.medin.2020.03.015

18. Patel BK, Wolfe KS, Pohlman AS, Hall JB, Kress JP. Effect of Noninvasive Ventilation Delivered by Helmet vs Face Mask on the Rate of Endotracheal Intubation in Patients With Acute Respiratory Distress Syndrome: A Randomized Clinical Trial. JAMA. 2016;315(22):2435-41. doi: 10.1001/jama.2016.6338

19. Hui DS, Chow BK, Lo T, Ng SS, Ko FW, Gin T et al. Exhaled air dispersion during noninvasive ventilation via helmets and a total facemask. Chest. 2015;147(5):1336-1343. doi: $10.1378 /$ chest.14-1934

20. Ferreyro BL, Angriman F, Munshi L, Sorbo LD, Ferguson ND, Rochwerg B et al. Association of Noninvasive Oxygenation Strategies With All-Cause Mortality in Adults With Acute Hypoxemic Respiratory Failure: A Systematic Review and Metaanalysis. JAMA. 2020;324(1):1-12. doi: 10.1001/jama.2020.9524

21. Associação de Medicina Intensiva Brasileira. Diretrizes Brasileiras de Ventilação Mecânica. [Internet]. 2013. Disponível em: https://bit.ly/2EFc9Za

22. Carvalho CRR, Toufen Junior C, Franca SA. III Consenso Brasileiro de Ventilação Mecânica. J Bras Pneumol. 2007;33(Supl 2):54-70.

23. Shang $Y$, Pan $C$, Yang $X$, Zhong $M$, Shang $X$, Wu Z et al. Management of critically ill patients with COVID-19 in ICU: statement from front-line intensive care experts in Wuhan, China. Version 2. Ann Intensive Care. 2020;10(1):73. doi: 10.1186/s13613020-00689-1

24. Sartini C, Tresoldi M, Scarpellini P, Tettamanti A, Carcò $\mathrm{F}$, Landoni $\mathrm{G}$ et al. Respiratory Parameters in Patients With COVID-19 After Using Noninvasive Ventilation in the Prone Position Outside the Intensive Care Unit. JAMA. 2020; 323(22):2338-40. doi: 10.1001/jama.2020.7861 